\title{
A Haptic-Audio Simulator Indoor Navigation: To Assist Visually Impaired Environment Exploration
}

\author{
Catherine A. Todd and Katy Naylor
}

\begin{abstract}
VirtuNav is a haptic-, audio- enabled Virtual Reality (VR) simulator that facilitates persons with visual impairment to explore a 3D computerized model of a real-life indoor location, such as a classroom or hospital. For administrative purposes, the screen displays a 2D overhead view of the map to monitor user progress and location relative to the reconstructed 3D environment. The system offers two unique interfaces: a free-roam interface where a user can freely navigate and interact with the model, and an edit mode where an administrator can manage test users, manage maps and retrieve test data. VirtuNav is developed as a practical application offering several unique features including map design, semi-automatic 3D map reconstruction and object classification from 2D map data. Visual and haptic rendering of real-time 3D map navigation are provided, and automated administrative functions including determination of shortest path taken, comparison with the actual path taken, and assessment of performance indicators relating to time taken for exploration and collision data. VirtuNav is a research tool for investigation of user familiarity developed after repeated exposure to the indoor location, to determine the extent to which haptic and/or sound cues improve a visually impaired user's ability to navigate a room or building with or without occlusion. System testing reveals that spatial awareness and memory mapping improve with user iterations within VirtuNav. The application is for greater real world engagement: to build confidence in real world experiences, enabling persons with sight impairment to more comfortably and readily explore and interact with environments formerly unfamiliar or unattainable to them.
\end{abstract}

Index Terms-Virtual environment, indoor navigation, haptics, 3D reconstruction, visually impaired.

\section{INTRODUCTION}

As estimated by the World Health Organization, in 2010 there were approximately 285 million people around the world who were sight impaired; those whose vision is limited or occluded to some extent, of which 39 million were blind [1]. According to recent sources [2], the incidence of visual impairment is on the increase, with a 9\% rise from 2006 to 2014 of children registered with partial sight or blindness.

Visual impairment and restriction of environment contributes to loneliness, depression, social isolation [3], [4] and activity and participation restrictions [3], [5], [6]. Almost $50 \%$ of visually impaired persons feel 'moderately' or 'completely' isolated, socially and environmentally [7]. Less than $25 \%$ are offered mobility training to assist in independent

Manuscript received August 3, 2014; revised October 22, 2014.

C. A. Todd is with the Faculty of Engineering and Information Sciences, University of Wollongong in Dubai, Dubai 20183, UAE (e-mail: catherinetodd@uowdubai.ac.ae)

K. Naylor is with Moorfields Eye Hospital Dubai, Dubai 20183, UAE (e-mail: katy.naylor@moorfields.ae). travel [8] and around 15\% claim to not participate in leisure activities external to their home [9]. At least $30 \%$ of visually impaired persons do not independently travel outside their homes [6]. Further, blind people will follow familiar or known routes when moving between locations, due to the stress and anxiety caused by exploration [6]. Fear and uncertainty are perceptual difficulties that inhibit the mobility of those with sight impairment [6], [10]. There is much greater risk of unintentional injury during exploration, such as falling or collision related accidents, compared with sighted individuals [11]. A visually impaired person must frequently overcome the challenges of navigation between locations [6], [12], with both physical [11] and perceptual barriers [10].

Those who are significantly impaired or blind may use navigation aids for mobility, such as walking sticks or guide dogs; the former predominantly when there is significant loss of peripheral vision to detect objects away from one's central vision. Users with minimal central vision loss will experience little trouble with object identification or perception of surrounding obstacles. Several assistive devices are available [6], such as handheld GPS's, yet navigation remains potentially dangerous due to unfamiliar obstructions such as chairs and tables as well as hazardous areas including staircases and ledges [13]. An identified increased risk of social isolation and avoidance of exploring new environments due to sight impairment exists. Assistive technologies can help bridge this gap.

The proposed solution addresses the difficulty associated with indoor navigation for greater social and environmental engagement, to help those with visual impairment by providing a support tool that offers haptic (force) feedback and audio prompts as navigational aids. The software facilitates semi-automatic recreation of a real-life environment in 3D virtual space, offering a support system for indoor exploration, for the user to attain heightened spatial awareness and gain a better understanding of their surroundings in which they are immersed. The user moves through and interacts with the environment using a haptic device and keyboard. The system tracks user progress as they navigate through the virtual indoor space and around embedded objects. The software provides visual and haptic feedback as well as audio cues, representing the objects and associated collision by the user, in 3D. A virtual environment (VE) provides a safe space for users to familiarize themselves with the location before visiting it in physical presence, helping to overcome physical challenges and fear of accident-related injury. VirtuNav provides navigational assistance and an accurate representation of real world indoor locations in a VE, with objective and subjective validation.

Targeted system users are those with almost complete 
central vision loss. Children and teenagers may prove a more receptive participant demographic as they are quickly adaptable to new technologies as opposed to the elderly; to use and interpret haptic feedback faster, adapting more quickly to the application. To provide a safe, seamless integration to the system, a VE has been created for the user to explore that reconstructs the natural world.

Technological advancements have been made in the research to address visually impaired navigation using VR schemes. However, a 3D, semi-automatic, customized approach that efficiently extracts and reconstructs map data, as an assessment and guiding tool in VR, is lacking. The existing research and identified deficit are examined in the following section. The remainder of the paper presents the design and implementation, testing and findings of the application to address this technological and research deficit through development of an assistive technology for exploration and wider environmental engagement, less mobility restriction and towards reduced social isolation.

\section{LITERATURE REVIEW}

Several studies examine the extent to which fear and uncertainty inhibit visually impaired persons to engage with their environment [5], [6], [10], leading to social isolation and subsequent health issues including depression [3], [4]. In a study examining elderly, sight impaired groups (above 70 years old), research findings revealed visual impairment to be 'a significant risk factor for additional medical conditions, activity limitations, and participation restrictions' [5]. In an in-depth literature review, [6] concludes that improving mind mapping of visually impaired persons via enhancement of way finding and orientation skills leads to improved quality of life, mobility and independence. 3D immersive, interactive technology that is customized to map data is lacking but may assist in heightened spatial awareness, to overcome perceptual and physical barriers that restrict mobility. The research challenge of constructing such an environment to assist in mind mapping is identified.

Existing research initiatives have developed devices and/or software solutions that cater to the needs of the visually impaired for determination and navigation of a location, of which [14], [15], and [16] focus on outdoor navigate on using handheld devices. Two VR research-based solutions that focus on indoor navigation include BlindAid [17] and the Kulturhuset Prototype [18]. The BlindAid system [17] consists of a VE replica of a real life environment where the scenario is a single, horizontal level such as a floor of a building. This system applies haptic feedback and audio cues to test the user's ability to navigate the environment as well as how comfortably and accurately they move about the corresponding real world environment [17]. The user moves through the VE using the haptic device and receives force feedback when the avatar, represented as a moving dot on the screen, comes into contact with an object in the VE. As reviewed by Lahav [19], haptic feedback provided is similar to that of the forces exerted during use of a long cane. The system is comprised of three interfaces: one containing the VE through which the user navigates, an evaluation interface for the purpose of recording a user's behavior and an editor mode which enables one to create the VE by importing AutoCAD files into the system [19]. During the system's usability testing, participants preferred the VE to provide clear spatial information and to reserve unique textures for designated areas (such as stairs), as opposed to complicated textures which confused users [19].

In Stockholm, a program was developed as part of a thesis study to observe the impact of haptic and audio on VE navigation [18]. The 3D reconstruction that is haptic- and audio- enabled was modeled on a real-life location in Stockholm known as Kulturhuset [18]. The user navigated about the VE using the PHANToM Omni (3DoF) haptic device, representing a virtual walking stick. Some of the techniques implemented in the Kulturhuset prototype included magnetic force reflection for collision response and simulation of environmental sounds. Audio feedback associated with a collided object invoked a sound file, either in the form of speech or non-verbal cues. Magnetic force was attached to a virtual escalator and upon moving close to the escalator, the avatar attached to the force until the user reached the second floor, at which point the force was disabled. During the testing phase, many users found it difficult to identify and use this magnetic force appropriately until informed about it by the administrator [18]. The volume of an environmental sound was increased or decreased based on the user's distance from the object producing the sound, and many users found that this feature helped them navigate through the VE and find specific objects more easily [18].

Research findings reveal the utility of computer-based interactive simulations to assist in visually impaired user navigation to achieve heightened environmental familiarity, yet a 3D haptic-, audio- enabled, customized environment based on selectable image map data is lacking. This work addresses this research challenge. Further, through testing and validation, the work considers the extent to which exposure to the simulation improves familiarity and mind mapping capabilities for visually impaired navigation within an indoor environment. The social impact of this technology is towards improved spatial awareness within an unfamiliar location to build confidence and reduce perceptual and physical barriers, for a higher incidence of exploration and hence greater environment and social engagement.

\section{MethodOLOGY}

Software design considerations identified key features for enhancing visually impaired navigation via simulation. Pre-knowledge of the anticipated route and landmark occurrences are advantages to navigation and a means to access and aid mobility performance [6], [10]. Abstract concerns such as safety or getting lost, as well as the construction of mental maps relating to perception of difficulty [10] are also important considerations. Image-guided solutions can provide specific features or object occurrences to build confidence and reduce hesitation when interacting with the environment [10]. Sound cues can provide signals for specific objects to alert the user to their presence in the environment, for hazardous areas such as stairs, escalators [10]. Reliance on haptic and audio cues, as well as the creation of cognitive maps are important concerns 
for visually impaired navigation [6]. Map recognition provides measurement of configurational knowledge [6]. Tactile and audio maps given to sight impaired users and sketch map interpretations provide suitable testing platforms for assessment of cognitive mapping ability [6], as do creating models or walking an inferred route [20]. To address the key considerations and contribute toward the literature research findings, a practical, low-cost assistive application based on a real life indoor location for visually impaired users to explore has been developed. The system design is shown in the following diagram (Fig. 1).

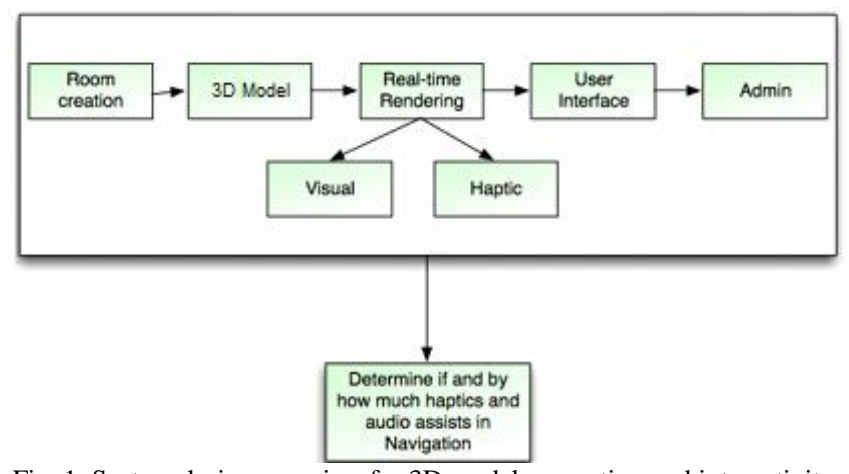

Fig. 1. System design overview for 3D model generation and interactivity.

First, a floor plan represented by image data is input to the system and wall boundaries, as well as objects, are semi-automatically extracted and classified, from the image. The 3D visual model is rendered from the extracted 2D objects, using distance dimensions input from the user and applied to the environment. Force feedback and visual rendering are applied to the model and delivered back to the user via the GUI and haptic interface, enabling user manipulation and interactivity with the environment. Sound cues are also applied. Administrative functions such as calculation of actual versus shortest path for determining user performance are implemented.

The system tracks user progress as they navigate through the virtual indoor space and around objects such as furniture. The software provides adequate visual and haptic feedback, and audio cues, to represent the objects and associated user collision in 3D. Through testing and validation, appropriate haptic and audio feedback are achieved at acceptable user discernment, to reflect object interactions as they would occur in reality and assist in object identification. Since a VE provides a safe space for users to explore a location before visiting it in reality, the software offers navigational aid and reconstruction of real world facilities and embedded obstacles Tests were conducted for system verification, validation and to determine the extent to which VirtuNav assists a user with indoor navigation and location familiarity.

Two independent parties interact with the system: users, who explore the VE directly and administrators, who manage user information and obtain results. Administrators can create or delete users from the system, retrieve information about user progress and generate maps for the users to interact with. $\mathrm{He} / \mathrm{she}$ can manage user profiles, adding detail such as percentage of visibility in each eye, a username and password, gender and age. Details can be used later to customize one's result query, as well as for research analysis. They can query the software based on a number of selected parameters, such as visibility range, age group, a specific user or a collection of users. Tabulated and graphical results are provided. They have the ability to create the maps that will be explored by the users. This is examined further in Section IV.

The visually impaired user's role is to navigate through the VE and interact with embedded objects. The VE is a 3D reconstruction of the map selected by the administrator. The user interacts with the VE and objects using a Novint Falcon 3DoF haptic device supported by keyboard functionality. The user can move forward, back and sideways within the VE, as well as rotate 45 degrees to the left or right. Sounds are emitted by certain objects in the VE, at different volumes depending on the distance. As the user navigates, object collisions trigger a force response through the haptic device paired with an audio response. The user may hear a sound identifying the name of an object by pressing a button.

Models of standard indoor objects such as tables and chairs are created and stored, for access and placement upon environment reconstruction. A map source represented by image data are input to the application and boundaries of the environment, such as room perimeter, as well as the locations of objects within it are stored in the form of coordinates. These coordinates are referenced and boundaries of the VE are generated automatically by the software.

Haptic and audio cues are associated with VE objects, modeled on their real-world properties. The real environment represented by the map source is assumed as static, with all embedded objects accurately mapped to the digital map source received. Dynamic aspects such as human movement are not mapped to the digital map source as they do not aid in environment familiarity. Force feedback simulates rigid body interactions which are suited to object interactions at the macro-level, as evident from the findings of Lahav [14]. Users indicated that an object's micro-level haptic properties are not useful in object detection or recognition [14].

Embedded processes were semi-automatic, for data acquisition, registration, and object and wall reconstruction. Automating the map generation process reduced administrator time spent in preparation for user testing, facilitating faster generation of results. Line segment recognition enabled classification of wall boundaries or objects automatically. Algorithms that provided room scale estimation from raster data to metric units were also included in the automation process. To optimize rendering time, realistic textures were not applied to objects. Similarly, fine haptic feedback was not simulated, preventing the user from being overwhelmed with too much stimuli. User-prompted voice-over identification is included to assist in user navigation, providing another feature for object identification in addition to simple haptic feedback. Simplified environmental sounds were added for enhanced user immersion and orientation. From Schloerb's findings [17], the use of background sounds helped users remain oriented in the $\mathrm{VE}$ and as such, are included in this application.

\section{IMPLEMENTATION}

\section{A. Floor Plan Segmentation and Object Classification}

To produce a $3 \mathrm{D}$ room reconstruction, a $2 \mathrm{D}$ map was input 
to the system (as an image) and the walls as well as embedded objects of the room are extracted and classified. The coordinate extractor class converts the image floor plan input to a set of line segment coordinates, via thresholding. The coordinates as output from this algorithm are used to create line segments which represent the reconstructed floor plan. An interface for the classification of lines and placement of additional furniture objects is presented to the administrator, comprising three screens. The first screen (Fig. 2) allows the administrator to group line segments together to represent walls or objects by clicking on the desired line segments and classifying them under a "tag" from a drop-down menu. The administrator is also asked to set a real-world value for a single line segment. Using this value, the program calculates a scale to be applied to calculate the real-world values for the remaining segments. The second screen (Fig. 3) allows the administrator to place additional furniture by selecting the desired object and clicking within the floorplan to generate a bounding box representing the object. The third screen (Fig. 4) allows the user's default starting position and orientation, at the center of the image facing north, to be modified.

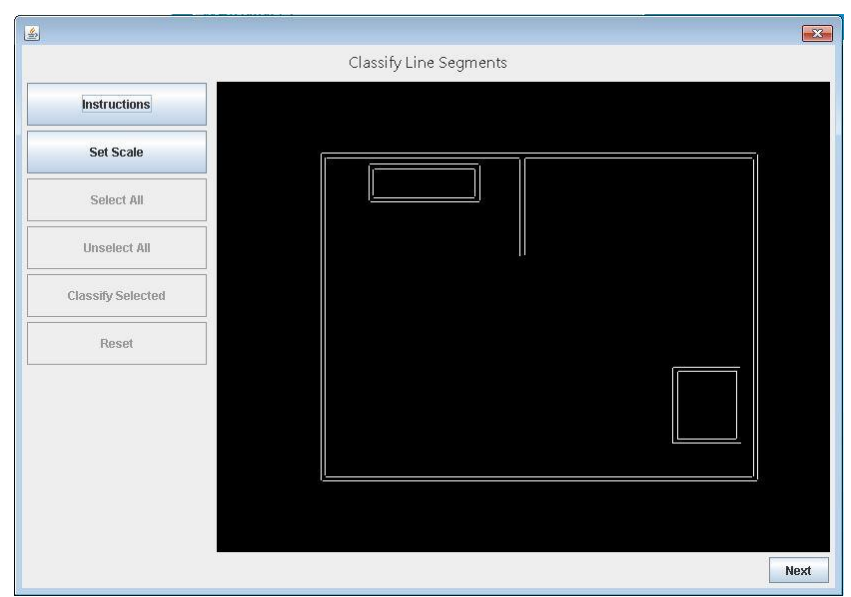

Fig. 2. User Interface for classification of existing line segments.

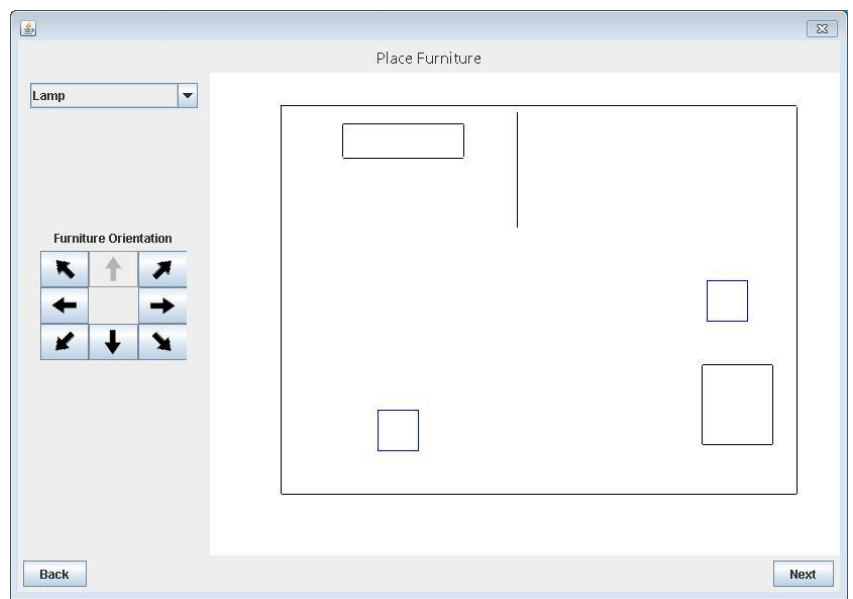

Fig. 3. User Interface for optional placement of additional furniture objects.

\section{B. 3D Reconstruction of the Indoor Scene}

The map (Fig. 5) is recreated in the software x3D using classified line segments and any placed objects. x3D uses the center of an object, its rotation or orientation, its position, and its size to reconstruct it in 3D. For example, a box may be created: $\quad "<$ Box $\quad$ size $=\quad \mid "$ "'+walls.get(j).length()+" "+height+" "+width+"|"/>". The wall is created using the
'Box' tag which requires $\mathrm{x}, \mathrm{y}, \mathrm{z}$ values which represent the element length, height, width. The $x$ value is attained through the length() function in the custom LineSegment class, which is the length of the line or the distance between two points, the $\mathrm{y}$ value is height and $\mathrm{z}$ value represents thickness. These values are predefined and calculated at the back end. The predefined height value of walls is calculated by dividing 3 by the meters per pixel calculated by the segmentation and classification process; the thickness of the walls is calculated by dividing 0.1559 by the meters per pixel. According to Meyers-Levy [21], the standard ceiling height is $3 \mathrm{~m}$ with the standard thickness of a plaster wall is $0.1559 \mathrm{~m}$.

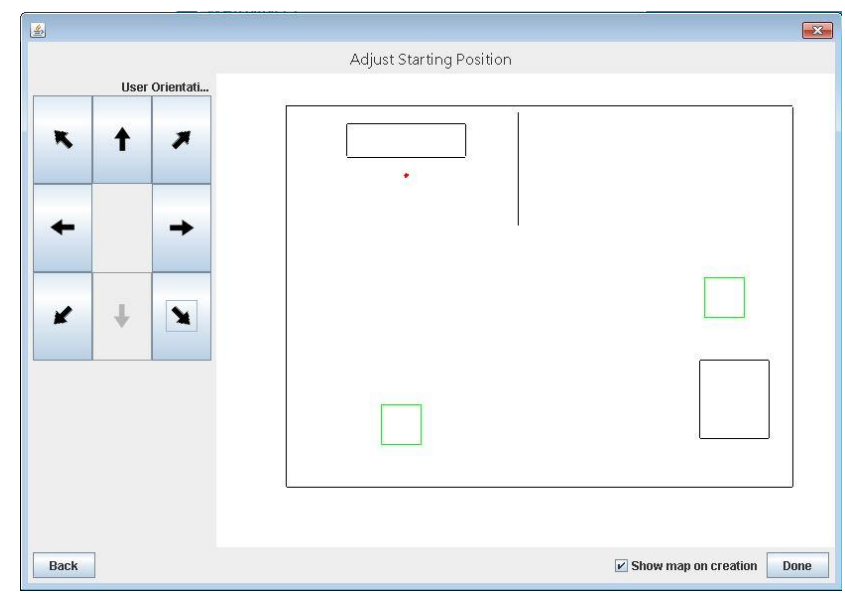

Fig. 4. User Interface for selecting a user starting position and orientation.

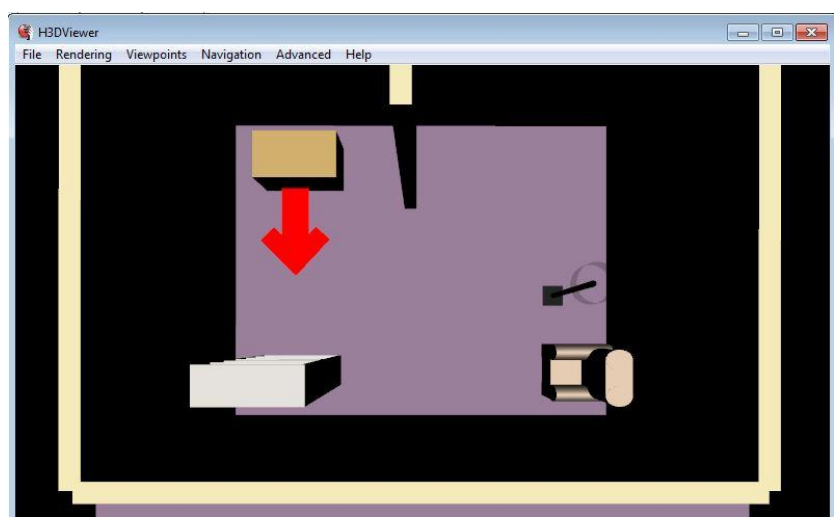

Fig. 5. 3D recreation of a map in $x 3 \mathrm{D}$ (top-down view). The red arrow represents the user's starting position and orientation.

Furniture such as chairs and tables are created as separate $\mathrm{X} 3 \mathrm{D}$ files. If certain line segments are classified as furniture, the appropriate furniture model is added to the map using the $<$ INLINE > node. The furniture is made on a 1 unit (1 meter) scale and as such requires scaling to fit the dimensions of the map. The aforementioned meters per pixel value is used to scale each furniture model before they are placed in the map. The 'Furniture' class is used to retrieve the height and location of the model to be placed appropriately in the map. Audio files and event handling are written within each model and therefore all instances of the models will react similarly. A transparent dummy box is created for the sole purpose of passing the aforementioned meters per pixel value to a python file. Once received, the python file saves this value and adjusts the user's walking speed based on the value. Using the $<$ Sound $>$ node, background or ambient noises are placed in the map as well as a footsteps sound effect. The footsteps location is bound to the location of the user to ensure that it is 
heard wherever the user travels. The <ROUTE> node is utilized which allows the dynamic passing of values between two nodes. In this instance, the position of the source of the footstep sound effect is bound to the position of the <ViewPoint> node, or camera, defined earlier in the construction.

\section{Haptic Rendering of the Scene and Event Handling}

The Novint Falcon, popular for simulating realistic force feedback in video games, constitutes the haptic interface. Within the haptic response, bumpy or smooth surfaces in textures are simulated. Users can interact with these surface types using the $3 \mathrm{DoF}$ device, to differentiate between various surfaces, such as discerning variability in surface hardness to assist with object identification.

Visual and haptic rendering algorithms are implemented using a variety of languages and API's that facilitate fast development and real-time response times, including H3DAPI, X3D and Python. H3DAPI allows for the use of $\mathrm{C}++$ and OpenGL for visual and haptic rendering for advanced programmers and upon installation, offers rH3DViewer and H3DLoad for real-time rendering. Further, the solution offered ease of event handling through Python scripting and capabilities for fast haptic rendering by H3DViewer and H3DLoad. A combination of X3D, an XML based file format to display graphics, and Python are used for ease of graphics creation and event handling. Several $<$ PythonScript $>$ nodes are placed to allow for event handling using Python scripts. Several $<$ ROUTE $>$ nodes are also added to pass values from nodes to the Python code and vice versa. A python file triggers audio cues when the stylus of the haptic device (or the proxy) comes in contact with a furniture object in the 3D VE. A different python code, the logger, is used to capture the location of the camera as the scene updates as well as the forces on the proxy to detect collision, to create a log of the user's progress. When a positive force is detected on either $\mathrm{x}, \mathrm{y}$, or $\mathrm{z}$ components of the proxy, the location of the collision is written in to the log file. Finally, a python code, the camera controller, is used to capture keyboard input and the location of the haptic device to move the camera about the VE. When an arrow key is pressed or the correct number is pressed on the numerical pad of the keyboard, the camera is moved by applying a forward vector on the identity matrix of the location of the camera. Other keys allow the camera to move backwards, move sideways and also rotate. If a collision is detected by the proxy, any forward movement of the camera is stopped until the collision is resolved. Footstep sounds are triggered when the camera moves forwards or backwards.

\section{Shortest Path Graph Creation}

After the user has explored the environment, it is of interest for the administrator to compare the actual path traversed with a shortest possible path, to examine variance. A shortest path algorithm has been constructed to determine that which the user could have taken during map exploration. The starting position, map width and length as well as a collection of classified line segments are taken as input to the algorithm. The greater measurement of the width and length is divided by a constant integer $\mathrm{N}$ to divide the map into an $\mathrm{N} x \mathrm{~N}$ grid. To ensure that the entire map is divided evenly, the modulus of the greater of the width and length is added to the quotient when divided by N. A graph is then created by creating a node (a square with side length of the quotient calculated) from the indicated starting position of the user within the map which serves as the root of the graph. The graph serves as a collection of bidirectional nodes that represent areas where the user is able to walk within the map.

The recursive algorithm begins with the root node and parses through each of the 8 possible surrounding nodes for validity. As each node is created (clockwise from the left of the current node), the algorithm ensures that: 1. the node exists such that its center is not outside of the map boundaries, 2. that the node does not intersect with any of the classified line segments, and 3. that the node has not been previously classified as invalid. If the node is valid, it is connected to the current node. The evaluation continues for all 8 possible nodes the user can move to from the current node. The children nodes of the current node are interconnected to one another. The current node is deemed complete by setting a flag to true and the recursive algorithm is called on the current node's children clockwise from the left (the left node, top left node, top node, etc.). The recursive algorithm terminates if the node it receives is NULL (an invalid node) or has already been evaluated (as indicated by the flag described formerly).

\section{E. Path Finding within the Map}

To determine the actual path taken by the user within the map, an algorithm has been implemented. A collection of points is input and regarded as points of interests in the map. These points of interest, including the starting positions, are the locations required of the user to reach as part of a quantitative analysis for their degree of learning. Each point of interest is mapped to the closest node in the generated graph; excluded is the starting position, which already belongs to the root node. This is determined by calculation of the Euclidean (straight line) distance between the given point and the center of each valid node. With this algorithm, even points located within invalid areas are mapped to the closest possible valid node.

Two path finding algorithms were considered to determine the optimum path between every node of interest: the $A^{*}$ path finding algorithm and the Jump Point Search path finding algorithm, which is an extension of the $\mathrm{A}^{*}$ algorithm. The $\mathrm{A}^{*}$ path finding algorithm considers both the cost of going to a node, expressed as a function $\mathrm{G}$, from the origin and a heuristic value, expressed as a function $\mathrm{H}$, from the node to the target [22]. The combined value, expressed as a function F, determines if a node will be part of the optimal path from the origin node to the target node [22]. In the Jump Point Search algorithm possible nodes are pruned depending on the direction from the origin node to the target node [23]. This system of pruning allows a quicker derivation of the optimal path compared to the performance time of $A^{*}[23]$. The $A^{*}$ algorithm has been implemented to generate the shortest path between several nodes of interest since the path finding within a generated map occurs in the administrative view since the process does not need to run in real-time for graphical and haptic rendering of the VE.

\section{F. Path Comparison}

Each time the system undergoes visual re-rendering, the 
position of the user is logged to a file. This file is opened for comparison and each point is mapped to the nearest valid node within the graph. Based on the administrator's chosen points of interest, a sub-path is derived from the user's complete path where the user starts from one point of interest to the next or until the end of the path (in cases where the next point of interest is never reached). To compare the user's sub-path and the shortest path from the selected points of interest, the Euclidean distance must be calculated. For this, the paths must be converted to scalar values indicating the distance between two nodes in the horizontal and vertical direction. If the number of resulting scalars for both paths are not equal, the path with the greater number of vectors is minimized by dividing the total number of scalars $(\mathrm{T})$ by the target number of scalars $(\mathrm{S})$, giving value $\mathrm{N}$ and modulus $\mathrm{M}$. The first (S-1) scalar values will be the sum of the next $\mathrm{N}$ scalars from 0 , and the last scalar value will be the sum of the next $\mathrm{N}$ scalars added to the last $\mathrm{M}$ scalars. With both paths having an equal number of scalars, the Euclidean distance for each pair (one scalar from each path) is calculated: first, the difference between the two scalars is calculated $(\mathrm{V})$ and then multiplied to obtain the transpose (square of $\mathrm{V}$ ), giving $\mathrm{D}$. The square root of $\mathrm{D}$ is added to the total sum. The total sum of the Euclidean distances is averaged by the total number of scalars of either path, to provide the final value.

\section{TRAINING Modules}

Various user training modules were developed for system familiarization and testing, to introduce various components, from haptics device use to 3D navigation.

Haptic Module: The purpose of this module is toward user familiarity with the haptic device, haptic feedback, 3D sensation and various 3D shapes. The user moves the device, which in turn moves the stylus on the screen. The user is asked to identify the shape or texture by exploring them with the haptics device. For haptic familiarity, the first and second lessons task the user to touch a cube and to identify three different, curved shapes, respectively: a sphere, a cylinder and a cone. The third lesson tasks the user to identify the surface texture of three cubes and a spring effect on a fourth cube for discernment of frictional surfaces and spring effects, toward usability and pleasantness.

Audio Module: This module allows the user to familiarize themselves with different types of audio cues, such as footsteps for movement, collision sounds and ambient noise. The user moves the stylus and upon collision with an object on the screen, the playback of an audio cue results. The administrator switches between various audio prompts and checks if the user can identify each one. A Python file detects the collision and plays the audio based on the type selected by the administrator at the front-end.

Movement Module: This module allows the user to familiarize themselves with movement controls. These movement controls are mapped to three sets of keys: the number keypad, the QWERTY keypad and the arrow keys. In this lesson, the user may try out the movement controls and select their preferred set of keys. A Python file handles the user's changes in position when the keys are pressed.

Integration Module: This module combines all of the aforementioned modules into a haptic and audio-enabled VE. The user is asked to navigate through this fully integrated VE. A speech component has been created, extended from the Speech 0.5.2 module which uses the built-in Windows speech synthesizer to convert text to audio. A string can be passed to this module in order to be read out by the speech synthesizer; such as speech.say("Hello") which would cause the speech synthesizer to say "Hello". When the " $\mathrm{t}$ " key or " 0 " key is pressed, the name of the object last collided with is read.

\section{TESTING}

This section outlines both usability and functional testing, for application verification and validation. In total, seven tests were conducted, including qualitative testing via surveys and quantitative testing, through capturing the position of the user over time, number of collisions and location of collisions. User ages varied from 9 to 55 years and their anonymity was retained throughout testing. Test conditions were safe and testing procedures were explained to each participant. Participants were sighted although blindfolded throughout the entire testing procedure, simulating late-onset blindness and test subjects had no priori knowledge of the testing environment. In his literature review of cognitive mapping of visual experiences for comparison between congenitally blind, late-onset blind and sighted persons, Ungar [20] determined that in many of the case studies he examined, congenitally blind participants were found to perform spatial tasks at the level of sighted participants, such as in tests of spatial inference and 'that lack of visual experience does not prevent the acquisition of spatial representation'. Further, in most studies research findings determined that early-blind participants performed to the level of late-blind and sighted participants in tasks that involved spatial memory [20]. In cases where early-blind participants performance was worse, it is determined that differences may be due to behavioural or coding strategies that provide a tendency rather than an inability toward specific spatial performance [20].

\section{A. Test 1}

Prior to the user undergoing haptic navigation testing in the simulated or real indoor location, each user underwent a series of training modules to help them become familiar with both the haptic device and movement around a VE. A usability survey was conducted to assess their performance and level of comfort within these training modules. Twenty-one users were tested within this section, with the following statistics: ages ranged between 9 and 55 years old. Male:female gender ratio of $52.38 \%: 47.62 \%$. User feedback determined that simple shapes were easy to identify, while complex shapes were difficult. Users commented that adding audio and frictional surfaces to objects could make them easier to identify and possibly help to tell objects apart from the boundaries of the haptic device. Users also suggested that audio may help more than frictional surfaces. Users concluded that existing movement control options were satisfactory and intuitive to learn.

\section{B. Test 2}

Users from Test 1 were then asked to explore a simple map 
without furniture (Room 1) and then provided with a usability survey on their interactions with the environment. Eight users were tested within this section, with the following statistics: ages ranged between 14 to 37 years old. Male:female gender ratio of $50 \%: 50 \%$. Based on survey results, users found it easy to locate the walls, and generally easy to follow along them. The speech component, for identifying the last object hit, was deemed helpful. Assumptions for Test 1 were validated; audio cues proved helpful in user discernment of objects from walls.

\section{Test 3}

Users were guided by the administrator through a virtual room to see whether they became more familiar with the room progressively, over the series of iterations. The first iteration was carried out by eleven users, the second iteration by six of the eleven users, the third iteration by three out of the six users and the fourth iteration by two out of the three users. Their performance was measured by tracking the amount of time spent in the room and the number of collisions over the number of room iterations (Fig. 6, Fig. 7). Upon conducting the user survey and analyzing results attained, it was determined that as the number of iterations through the map increases, the layout becomes more familiar. It was also apparent that when the user remembered the number of steps between room corners it reduced the time taken to make decisions regarding movement.

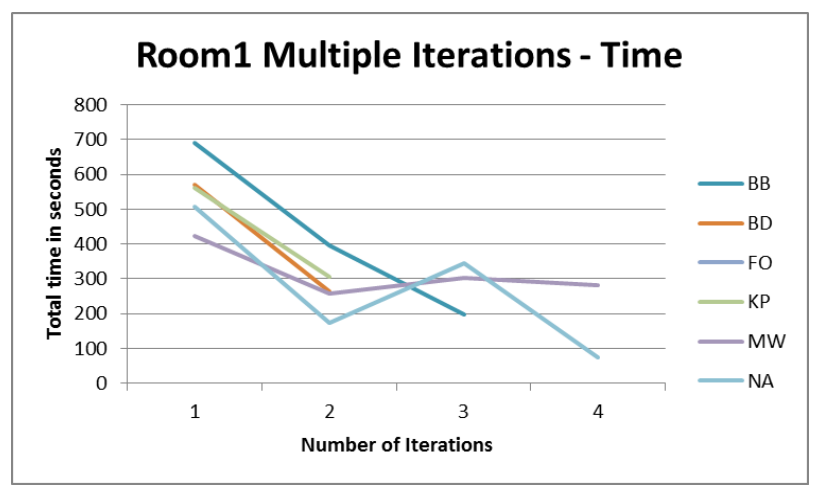

Fig. 6. Amount of time spent within map by users, for iterations 2 to 4 .

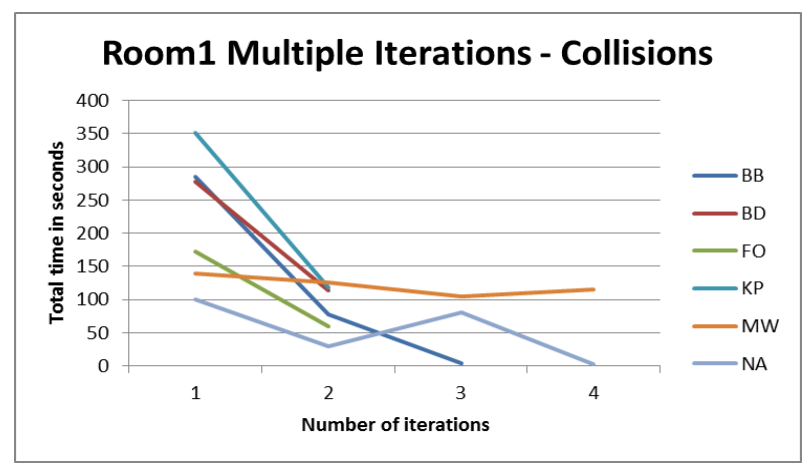

Fig. 7. Amount of collisions within map by by users, for iterations 2 to 4 .

\section{Test 4}

This test required users to find furniture pieces in the middle of the room. The user is first told that the objects are "...close to the door and north-east of the starting position." As the user explores the map, the user is occasionally told, after each successful rotation or pause, what direction the objects are relative to the direction the user is currently facing. Users can stop the test at any time to restart the map and to test estimated object positions. The goal of this test is to assess the user's mind mapping ability and spatial awareness to find new object locations relative to known object locations. After each successful test, the user is asked to draw the imagined location of furniture objects on a previously drawn sketch of the map. Four users performed this test with the following statistics: ages ranged between 14 and 37 years old; an average age of 27 years. Male-to-female gender ratio of 50\%. User feedback from the survey deemed that as the number of iterations through the map increased, the environment became more familiar. Further, remembering the number of steps between room corners reduced time taken for decision making.

\section{E. Test 5}

This test required users to move from one location to another, based on instructor commands. Users are instructed to travel to the following points under the agreed starting position of the user is in the bottom left corner of the room, facing north: the door, then the top right corner of the room, followed by the top left corner of the room and finally returning to the starting position. The goal of this test is to assess the user's mind mapping ability and virtual muscle memory to recall the location of these points. Four users undertook this test with the following statistics: ages ranged between 14 to 37 years old; an average age of 27 years. Male:female gender ratio of 50\%:50\%. From survey results it appeared that older users, aged 35-37, from total age range 14 to 37 years for all participants, are more comfortable following their mental map and virtual muscle memory to reach known destinations. However, even after becoming familiar with the virtual space, it was observed that participants are hesitant to stray away from familiar paths.

\section{F. Test 6}

This test required users to traverse the virtual room as far as possible while keeping the wall on their left. The user continued to move forward unless a corner was encountered and the user needed to turn. This test contains the same set of rules as Test 3 except the user does not attempt to traverse the room anti-clockwise. The user may stop the test at any time to gather and reinforce the mental map they have created and start over. The user may also explore the rest of the room to add details to their mental map. The goal of this test is to assess the user's mind mapping ability and memory when applied to a large map. At the end of the test, the user is asked to sketch the room as determined by their mind map. Three users were asked to perform this test with the following statistics: ages ranged between 14 to 37 years old; an average age of 28 years. Male:female gender ratio of $66.67 \%: 33.33 \%$. User feedback determined that it was difficult to traverse large maps and create mind maps of them. This was mainly due to the vast amount of information encountered, including the size of the room, shape of the room, number of steps taken, audio prompts, haptic prompts, keyboard usage and haptic device usage, and time-based location recall. Through practice, it was determined that users could learn to walk through the VE by taking one step at a time. Through repeated exposure to the VE, results indicate that users become more familiar with room shape and the necessary number of steps. It was evident that in-corners and out-corners can affect users' understanding of surfaces. 


\section{G. $\quad$ Test 7}

This test required users to traverse the physical counterpart of the room from Test 6 . Users were taken, blindfolded, to the physical location of the reconstructed class room. This was the same room as simulated in Test 6 . The user is handed a walking cane substitute: a plastic toy golf club. The user then proceeded to replicate Test 6 but within the physical location until they reach the location they have reached during their virtual experience. The users were filmed throughout the entire process. The goal of this test was to assess the user's mind mapping ability and recollection from a virtual experience to real life, to finally determine whether virtual navigation can assist in physical navigation, and to what extent. The same three users were asked to perform this test with the following statistics: ages ranged between 14 to 37 years old; an average age of 28 years. Male:female gender ratio of $66.67 \%: 33.33 \%$. User feedback revealed that objects could be anticipated and that the shape of the virtual room matched that of the real-life environment. However, the number of virtual steps and steps in real life were different, slowing down movement in the actual room as it was difficult to gauge its scale. The white cane substitute mimics the actions used with the haptic device when exploring virtually. Results revealed that navigation within the VE assisted in spatial awareness of the physical room, and objects within it.

\section{DISCUSSION}

The advantages of providing tactile maps and auditory cues of introducing a visually impaired user to an environment and to improve abstract level spatial thought as well as for mobility training are documented in the literature [6], [20]. Towards assisting in mental mapping for sight impaired persons during navigation, a virtual reconstruction of an indoor location with haptic and audio cues, derived semi-automatically from a 2D image-based floor plan has successfully been implemented and tested, proving to assist visually impaired users with indoor navigation. Implementation has been achieved using a pipeline approach. First, the system is able to automatically import an image floor plan and convert it in to a Portable Graymap Graphic which is sent to an algorithm to determine the line segment coordinates and saved into a dummy text file for further pre-processing. As a separate component, the system is able to draw line segments from a text file. The user can classify these segments as representing walls or furniture. Pieces of furniture can dynamically be added into the map. The user's starting position can be initialized in the same interface. The classified line segments are parsed and reconstructed in $3 \mathrm{D}$ under the $\mathrm{X} 3 \mathrm{D}$ format. Any classified furniture is derived from pre-created models and placed selectively within the scene. Haptic properties and Python support are added at this stage. Basic textures and environmental sounds are also added. The final result is presented to the user as a $3 \mathrm{D}$ virtual room with or without furniture, for navigation using the haptics device and audio prompts. All users tested were visually impaired: either blindfolded, obstructed or with natural visual impairment.

A novel, user-friendly and custom GUI has been created to enable a portal for using the application. The GUI integrates the processes into one single unit which allows administrators to provide an image file to create a 3D map. The administrator may also create or edit user profiles. A map may be selected from a list of pre-created maps and launched in the visual and haptic renderer. The user's location is tracked and stored in a log file, which can be late accessed by the administrator. Object collisions are stored in a separate log file that indicates the object hit and collision location. Both values are saved automatically and can be opened in the administrator dashboard. These values are used to calculate the length of time the user was in the VE, total number of collisions, as well as a visualization of the path the user took, with the original image set as the background, and the location of collisions, all stored in a database.

Test results for all participants, aged 9 to 55 in Tests 1 through 7, were collected, analyzed and examined to determine the extent to which haptic rendering and/or audio cues help a visually impaired user to navigate through the indoor location (virtually and in reality). Results of users' map traversals within the application are automatically stored into and retrieved from the database. Results show the total number of query results, the gender ratio of the test participants, their average age and average impairment values, as well as the specific performance of a user, including time for task duration and number of collisions, based on the maps they used, and the date and times of the day. Results are graphically displayed within the application in the form a pie chart for administrative purposes, to indicate the ratio of a user's object collision with various object constructs (such as chairs, tables and walls), a bird's eye view of the user's path in the map and a grid version of the map. The shortest path algorithm has been implemented and its development enables display on the grid. It is derived after selecting points of interest on the map via point-and-click. Users' traversals can then be compared with shortest paths.

Results of these tests indicate that a user's performance improves over time with exposure to the virtual indoor location using the VirtuNav application for navigation, both within the virtual room and for spatial awareness within the real (physical) location that has the same dimensions and construct as that which is recreated synthetically. Test results and user feedback highlighted the usefulness of the training module, the effectiveness and ease of use of the map creation and generation feature, and noted benefit to assist users in creation of a mind map, although further testing is warranted.

\section{CONCLUSION}

VirtuNav provides an assistive technological solution for the visually impaired to navigate an indoor location in VR, to become more familiar with both the synthesized as well as real location. A user interface for the administrator provides a portal to import and semi-automatically extract room data, objects and layout from a 2D map, for 3D room reconstruction. VirtuNav enables the user to explore this generated model through a user interface that provides visual, haptic and audio cues for navigation about the environment Unique features include map design, semi-automatic 3D map 
reconstruction and object classification from 2D map data, visual and haptic rendering of real-time 3D map navigation, and automated administrative functions including determination of shortest path taken, comparison with the actual path taken, and assessment of performance indicators relating to time taken for exploration and collision data. Test results indicate that user familiarity is developed after repeated exposure to the indoor location, revealing that haptic and audio cues can improve a visually impaired user's ability to navigate a room or building with or without occlusion. Spatial awareness and memory mapping were shown to improve with user iterations in the VirtuNav VE.

Implications of the research may help visually impaired children and adults more frequently and actively explore and engage with external locations outside their home, reducing levels of anxiety, loneliness, depression and social isolation. However, further testing is warranted. More testing can include a greater variety of age groups, extent and type of visual impairment and location variation, and comparison with a congenitally blind population. Future work should include questionnaires to gauge the degree to which participants find the application useful in overcoming perceptual and physical barriers associated with navigation, and subsequent social and health benefits.

\section{REFERENCES}

[1] World Health Organization (WHO). (2012). 10 facts about blindness and visual impairment. [Online]. Available: http://www.who.int/features/factfiles/blindness/index.html (accessed 05 March 2013).

[2] Blind Children UK. (2014). Childhood sight loss on the increase in the UK. [Online]. Available: http://www.blindchildrenuk.org/news/childhood-sight-loss-on-the-inc rease-in-the-uk (accessed 01 June 2014).

[3] R. Evans, W. Werkhoven, and H. Fox, "Treatment of social isolation and loneliness in a sample of visually impaired elderly persons," Psychological Reports, vol. 51, no. 1, pp. 103-108, 1982.

[4] C. O'Donnell, "Greatest challenge: vision loss and depression in older adults," Journal of Visual Impairment and Blindness, vol. 99, no. 4, pp 197-208, 2005.

[5] J. Crews and V. Campbell, "Health conditions, activity limitations and participation restrictions among older people with visual impairments," Journal of Visual Impairment and Blindness, vol. 95, no. 8 , pp. $453-467,2001$

[6] R. Jacobson, "Cognitive mapping without sight: four preliminary studies of spatial learning," Journal of Environmental Psychology, vol. 18, no. 3, pp. 289-305, 2005.

[7] T. Pey, F. Nzegwu, and G. Dooley, "Functionality and the needs of blind and partially-sighted adults in the UK: an interim report," Guide Dogs for the Blind Association, 2006.

[8] G. Douglas, S. Pavey, and C. Corcoran, "Access to information, services and support for people with visual impairment," Network 1000 - Access to Information, Services and Support, Vision 2020 UK and University of Birmingham, 2008.

[9] G. Douglas, C. Corcoran, and S. Pavey, "Opinions and circumstances of visually impaired people in Great Britain: Report based on over 1000 interviews," Network 1000 Report, Vision 2020 UK and University of Birmingham, 2006.

[10] O. Gustafson-Pearce, E. Billet, and F. Cecelja, "Perceptual impact of environmental factors in sighted and visually impaired individuals," British Journal of Visual Impairment, vol. 23, no. 1, pp. 25-30, 2005.

[11] R. Manduchi and S. Kurniawan, "Mobility related accidents experienced by people with visual impairment," AER Journal: Research and Practice in Visual Impairment and Blindness, vol. 4, no. 2, pp. 44-54, 2011.

[12] Arizona Office for Americans with Disabilities (AOAD). (2007). ADA resources: Living with blindness; physical assistance. [Online]. Available: http://know-the-ada.com/t5/ada-living-blindness.html

[13] S. Mau, M. Makatchev, and N. A. Melchior, "BlindAid: an electronic travel aid for the blind," Robotics Institute Report
[CMU-RI-TR-07-39], Pittsburgh, Pennsylvania, United States of America, 2008.

[14] O. Lahav, D. Schloerb, S. Kumar, and M. Srinivasan, "BlindAid: A learning environment for enabling people who are blind to explore and navigate through unknown real space," Virtual Rehabilitation, IEEE, August 25-27, 2008, pp. 193-197.

[15] K. Yatani, N. Banovic, and K. Truong, "SpaceSense: Representing geographical information to visually impaired people using spatial tactile feedback," in Proc. the SIGCHI Conference on Human Factors in Computing Systems, 2012, pp. 415-424.

[16] J. Sugiyama, D. Tsetserukou, and J. Miura, "NAVIgoid: Robot navigation with haptic vision," in Proc. SA '11 SIGGRAPH Asia 2011 Emerging Technologies, no. 9, pp. 12-15, Dec. 2011.

[17] D. W. Schloerb, O. Lahav, J. G. Desloge, and M. A. Srinivasan, "BlindAid: Virtual environment system for self-reliant trip planning and orientation and mobility training," Haptics Symposium, IEEE, pp. 363-370, March 2010.

[18] Y. Huang, "Design and evaluation of 3D multimodal virtual environments for visually impaired people," Doctoral Thesis in Human-Computer Interaction, KTH, Stockholm, Sweden, 2010,

[19] O. Lahav, D. Schloerb, S. Kumar, and M. Srinivasan, "A virtual environment for people who are blind - A usability study," Journal of Assistive Technologies, vol. 6, no. 1, pp. 38-52, 2012.

[20] S. Ungar, "Cognitive mapping without visual experience," in R. Kitchin ed., Cognitive Mapping: Past, Present, and Future, Routledge, London, 2000.

[21] J. Meyers-Levy and R. Zhu, "The Influence of ceiling height: the effect of priming on the type of processing that people use," Journal of Consumer Research, vol. 34, no. 2, pp. 174-186, 2007.

[22] P. Lester. (2005). "A* pathfinding for beginners. [Online]. Available: http://www.policyalmanac.org/games/aStarTutorial.htm

[23] D. Harabor. (2011). Jump point search. [Online]. Available: http://harablog.wordpress.com/2011/09/07/jump-point-search/

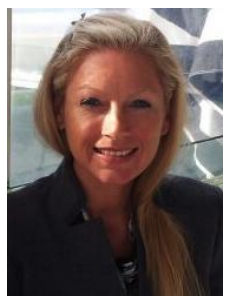

Catherine A. Todd received her PhD in electrical engineering from the University of Wollongong, Australia, accepted without modification in October 2006. In 2002 she was awarded a bachelor's in electrical engineering from the same university, with honors (class I). Her research specialization is in medical simulation, virtual manipulation, haptic and real-time visual rendering, and development of assistive technologies for special needs citizens.

She completed her work training in electrical engineering in 2002 after working with BHP as a degree cadet, Port Kembla Steelworks employed 1997-2002. She is currently employed as an associate professor in electrical engineering at the University of Wollongong in Dubai. She is the director of the Simulation and Smart Systems $\left(\mathrm{S}^{3}\right)$ Research Centre, which she established in April 2014. She has published in more than 30 publications, most with a high impact factor (IEEE, JLO) and ranked high in her research specialization

Dr. Todd is a professional member of IEEE and Signal Processing Society, and invited external referee for journals including the Int. Journ. Comp. Ass. Radiol. and Surg. (IJCARS), Pattern Recognition Letters, and Computer Animation and Virtual Worlds (CAVW)). She has received several teaching and research-based awards.

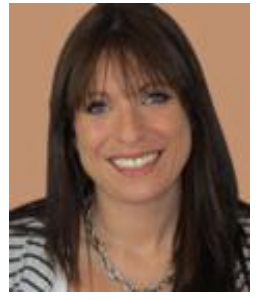

Katy Naylor is a practicing orthoptist at Moorfields Eye Hospital, Dubai. She graduated with a PGCE post compulsory sector (highly commmended) from the Institute of Education, London (2008-2010). She received her BMedSci (honors) in orthoptics 2:1 (2001-2004). Ms Naylor has extensive experience with all aspects of orthoptic work, including, strabismus and ambylopia, nystagmus, highly complex congenital and acquired ocular motility disorders, congenital and acquired orbital and adnexal disorders, congenital and acquired neurological conditions, congenital and developmental ocular abnormalities, genetic disorders and systemic diagnoses with associated ocular abnormalities.

She is the head of orthoptist, clinical specialist, education coordinator and lead clinical tutor at Moorfields Eye Hospital, Dubai (2011-current), originally from Moorfields Eye Hospital London (2004-2011). During her employment as clinical specialist at all Moorfields outreach centers in London, she was also an appointment lecturer at City University, University College London and Barts and the London School of Medicine.

Ms Naylor is fully registered and licensed with the Health Professionals Council in the UK. 